Georgian Mathematical Journal

Volume 13 (2006), Number 4, 687-691

\title{
NEW ESTIMATES OF THE SINGULAR SERIES CORRESPONDING TO POSITIVE QUATERNARY QUADRATIC FORMS
}

\author{
GURAM GOGISHVILI
}

\begin{abstract}
Let $m \in \mathbb{N}, f$ be a positive definite, integral, primitive, quaternary quadratic form of the determinant $d$ and let $\rho(f, m)$ be the corresponding singular series.

When studying the best estimates for $\rho(f, m)$ with respect to $d$ and $m$ we proved in [4] that

$$
\rho(f, m)=O\left(d^{-\frac{1}{3}} m \ln \ln b(d m)\right)
$$

where $b(k)$ is the product of distinct prime factors of $16 k$ if $k \neq 1$ and $b(k)=3$ if $k=1$.
\end{abstract}

The present paper proves a more precise estimate

$$
\rho(f, m)=O\left(d_{0}^{-\frac{1}{3}} d_{1}^{-\frac{1}{2}} m \ln b\left(d_{1}\right) \ln \ln b(m)\right),
$$

where $d=d_{0} d_{1}, d=\prod_{p \mid 2^{5} d} p^{h(p)}, d_{0}=\prod_{\substack{p\left|2^{5} d \\ p\right| 2^{2 m}}} p^{h(p)}, d_{1}=\prod_{\substack{p \mid 2^{4} d \\ p \nmid m, p>2}} p^{h(p)}, h(p) \geqslant 0$ if $p>2 ; h(2) \geqslant-4$.

The last estimate for $\rho(f, m)$ as a general result for quaternary quadratic forms of the above-mentioned type is unimprovable in a certain sense.

2000 Mathematics Subject Classification: 11E20, 11E25.

Key words and phrases: Singular series, quadratic forms, asymptotic formula, representation of numbers.

\section{INTRODUCTION}

Let

$$
f=\sum_{\alpha, \beta=1}^{4} a_{\alpha \beta} x_{\alpha} x_{\beta}
$$

be any positive definite, integral, primitive, quaternary quadratic form of the determinant $d=d(f)$, so the $\operatorname{gcd}\left(a_{11}, a_{22}, a_{33}, a_{44}, 2 a_{12}, \ldots, 2 a_{34}\right)=1$.

We consider the main term of formulas for the number of representations $r(f, m)$ of $m \in \mathbb{N}$ by $f$. The main term expressed by the so-called singular series $\rho(f, m)$ can be represented as an infinite product over all primes $p$

$$
\rho(f, m)=\frac{\pi^{2} m}{d^{\frac{1}{2}}} \prod_{p \geqslant 2} \chi(p) .
$$

The formulas for the $\chi(p)$ (even under more general assumptions) are obtained by Malyshev [6]. These formulas are simplified in some cases and represented in the convenient form in [1]. 
The estimates of $\rho(f, m)$ with respect to $d$ and $m$ are important for the investigation of the asymptotic behavior of $r(f, m)$, determination of one-class genera of the forms (1), the existence of the so-called Gauss type formulas for $r(f, m)(r(f, m)=\rho(f, m))$ and in other applications.

In the paper [6] some estimates of $\chi(p), p \geqslant 2$, are given. They yield

$$
\rho(f, m)=O\left(d^{\frac{1}{2}} m^{1+\varepsilon}\right)
$$

for any $\varepsilon>0$.

Studying the representation of numbers by sums of squares, Rankin [7] estimated the corresponding $\rho(f, m)$. Some analogous results for a quaternary form of special type are obtained by Kiming [5]. In [2] we essentially improved the existing results and obtained

$$
\rho(f, m)=O\left(d^{-\frac{1}{3}+\varepsilon_{1}} m^{1+\varepsilon_{2}}\right)
$$

for any $\varepsilon_{1}>0, \varepsilon_{2}>0$ and calculated the constant in the "O-term". This constant depends only on $\varepsilon_{1}$ and $\varepsilon_{2}$.

The papers [3] and [4] give more precise estimates

$$
\rho(f, m)=O\left(d^{-\frac{1}{3}} m \ln \ln d \ln \ln m\right)
$$

and

$$
\rho(f, m)=O\left(d^{-\frac{1}{3}} m \ln \ln b(d m)\right),
$$

where $b(k)$ is the product of distinct prime factors of the number $16 k$ if $k \neq 1$, and $b(k)=3$ if $k=1$.

The paper [4] gives an estimate for $n$-ary $(n \geqslant 5)$ quadratic forms too

$$
\rho(f, m)=O\left(d^{-\frac{n-2}{2(n-1)}} m^{\frac{n}{2}-1}\right) .
$$

The present paper sharpens the result (6) and proves

$$
\rho(f, m)=O\left(d_{0}^{-\frac{1}{3}} d_{1}^{-\frac{1}{2}} m \ln b\left(d_{1}\right) \ln \ln b(m)\right)
$$

where $d_{0} d_{1}=d, d_{0}=\prod_{\substack{p\left|2^{5} d \\ p\right| 2 m}} p^{h(p)}, d_{1}=\prod_{\substack{p \mid 2^{4} d \\ p \nmid m, p>2}} p^{h(p)}, h(p) \geqslant 0$ if $p>2$ and $h(2) \geqslant-4$.

The estimate (7) as a general result for quaternary quadratic forms of the above-mentioned type is unimprovable in a certain sense since the estimate $O\left(d_{0}^{-\frac{1}{3}} d_{1}^{-\frac{1}{2}} m\right)$ is not valid for any forms of such kind. An example of such extreme forms is constructed in [3].

\section{Notation and Some Preliminary Results}

It is known (cf., for example, [6]) that for any prime $p \geqslant 2$ and quadratic form (1) there exist integers $e_{\alpha}$ and quadratic forms $\phi_{\alpha}, \alpha=\overline{1, s}$, such that

$$
f \equiv \sum_{\alpha=1}^{s} p^{e_{\alpha}} \phi_{\alpha}\left(\bmod p^{e_{s}+3}\right)
$$


where $-1 \leqslant e_{1}<e_{2}<\cdots<e_{s}$ (if $p=2$, then any $n_{\alpha}$-ary $\phi_{\alpha}$ may be diagonal or of the type $\phi_{\alpha}=\sum_{\beta=1}^{n_{\alpha} / 2}\left(2 a_{\alpha \beta}^{\prime} x_{\alpha \beta}^{2}+2 a_{\alpha \beta}^{\prime \prime} x_{\alpha \beta} y_{\alpha \beta}+2 a_{\alpha \beta}^{\prime \prime \prime} y_{\alpha \beta}^{2}\right)$ and only then $e_{1}$ may be -1 . If $p \neq 2$, then $\phi_{\alpha}$ is diagonal and $\left.p \nmid \operatorname{det}\left(\phi_{\alpha}\right), \alpha=\overline{1, s}\right)$.

Let $p$ be any prime factor of $d$ (more exactly, $p$ be a factor of $2^{4} d$, since $d$ may be the number of type $2^{-4} d_{*}$ with $d_{*}$ being an odd integer), $d=p^{h(p)} d_{p}, p \nmid d_{p}$, $m=p^{w} m_{p}, p \nmid m_{p}, w=w(p) \geqslant 0$. According to the formulas for $\chi(p)$ (cf., [6] or [1]) we obtain the estimates of $\chi(p), p \mid 2^{4} d, p>2$. In all possible cases, for the representable $m$ and the forms (1) we have

$$
\begin{gathered}
\chi(p)=2 \quad \text { if } \quad w=0, \quad n_{1}=1 \\
\chi(p) \leqslant 1+\frac{1}{p} \quad \text { if } \quad w=0, \quad n_{1}>1
\end{gathered}
$$

so

$$
\chi(p) \leqslant 2 \text { if } w=0 .
$$

An estimate for $w>0$ is obtained in [2].

$$
\chi(p) \leqslant p^{\frac{h(p)}{6}}\left(1+p^{-2}\right)\left(1+p^{-1}\right) \quad \text { if } \quad w>0 .
$$

From the formulas for $\chi(2)$ (cf., [2]) we obtain

$$
\begin{aligned}
\chi(2) \leqslant 2^{e_{1}} & +\sum_{e_{1}<t \leqslant w+2} 2^{t-2-\sum_{n=1}^{l(t)} n_{\alpha}\left(t-e_{\alpha}-1\right) / 2+\nu(t)} \\
& + \begin{cases}2^{w-B(w+2) / 2+2.5-\nu(w+3)} & \text { if } 2 \nmid B(w+3), \\
0 & \text { if } 2 \mid B(w+3),\end{cases}
\end{aligned}
$$

where $\nu=\nu(t)=1$ if $2 \mid \sum_{\alpha=1}^{l(t)} n_{\alpha} ; \nu(t)=\frac{1}{2}$ if $2 \nmid \sum_{\alpha=1}^{l(t)} n_{\alpha}$,

$$
B(t)=\sum_{\alpha=1}^{l(t)} n_{\alpha}\left(t-e_{\alpha}\right), \quad l(t)= \begin{cases}0 & \text { if } t \leqslant e_{1} \\ k & \text { if } e_{k}<t \leqslant e_{k+1} \\ s & \text { if } t>e_{s}\end{cases}
$$

In a similar way as it was done in [2], from the last estimate we obtain

$$
\chi(2) \leqslant 4 \cdot 2^{\frac{h(2)}{6}} \quad \text { if } \quad w \geqslant 0
$$

where $d=2^{h(2)} d_{2}, h(2)$ and $d_{2}$ are integers, $h(2) \geqslant-4,2 \nmid d_{2}$.

\section{Estimates of $\chi(p), p \nmid 2^{4} d, p>2$}

Let $m=p^{w} m_{p}, p \nmid m_{p}$ and

$$
\delta=\left(\frac{d}{p}\right)
$$


be the Jacobi symbol. The paper gives the formulas for the corresponding $\chi(p)$ in the above-mentioned case.

$$
\chi(p)=\left(1-\delta p^{-2}\right) \sum_{0 \leqslant t \leqslant w} \delta^{t} p^{t} .
$$

It follows from the last formula that

$$
\begin{aligned}
\chi(p) & \leqslant 1+p^{-2} \text { if } p \nmid 2^{5} d m, \\
\chi(p) & <\left(1-p^{-2}\right) \sum_{t \geqslant 0} p^{-t}=\left(1-p^{-2}\right)\left(1-p^{-1}\right)^{-1} \\
& =1+p^{-1} \quad \text { if } \quad p \nmid 2^{5} d, \quad p \mid m .
\end{aligned}
$$

\section{Estimate of $\rho(f, m)$}

Now using (10), (8), (9), (11) and (12) we obtain

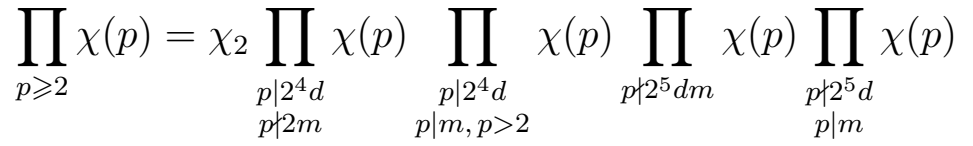

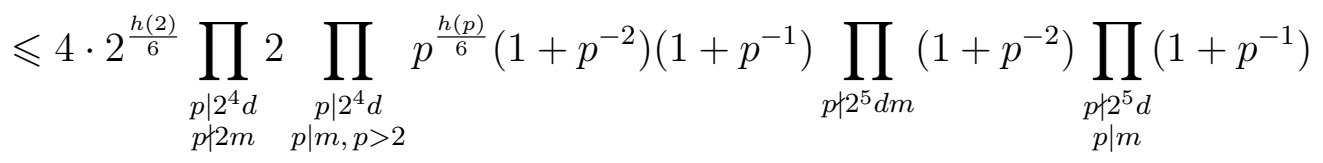

$$
\begin{aligned}
& \leqslant 4 \cdot d_{0}^{\frac{1}{6}} 2^{\sigma\left(d_{1}\right)} \prod_{p \geqslant 2}\left(1+p^{-2}\right) \prod_{p \mid m}\left(1+p^{-1}\right)
\end{aligned}
$$

where $\sigma\left(d_{1}\right)$ is the number of prime divisors of $d_{1}$.

It is obvious that $2^{\sigma\left(d_{1}\right)}<b\left(d_{1}\right)$, so $\sigma\left(d_{1}\right)=O\left(\ln b\left(d_{1}\right)\right)$.

Let $m>1$ and $p_{1}, \ldots, p_{\sigma(m)}$ be the first $\sigma(m)$ prime numbers, then using the well-known estimates (cf., for example, [8]) we obtain

$$
\begin{aligned}
\prod_{p \mid m}\left(1+p^{-1}\right) & \leqslant \prod_{2 \leqslant p \leqslant p_{\sigma(m)}}\left(1+p^{-1}\right)=O\left(\ln p_{\sigma(m)}\right) \\
& =O(\ln (\sigma(m) \ln \sigma(m)))=O(\ln \sigma(m))=O(\ln \ln b(m)) .
\end{aligned}
$$

This result together with the estimate

$$
\prod_{p \geqslant 2}\left(1+p^{-2}\right) \leqslant \sum_{a=1}^{\infty} a^{-2}=\frac{\pi^{2}}{6}
$$

lead us to the final result for the product

$$
\prod_{p \geqslant 2} \chi(p)=O\left(d_{0}^{\frac{1}{6}} \ln b\left(d_{1}\right) \ln \ln b(m)\right) .
$$

Clearly, (2) and (16) give the desired result (7). 


\section{REFERENCES}

1. G. P. Gogishvili, The summation of a singular series that is connected with diagonal quadratic forms with four variables. (Russian) Trudy Tbiliss. Mat. Inst. Razmadze 38(1970), 5-30.

2. G. P. Gogishvili, On an unimprovable estimate of singular series of positive quadratic forms. (Russian) Proc. A. Razmadze Math. Inst. 109(1995), 41-51.

3. G. Gogishvili, On the general unimprovable estimates of the singular series of positive quadratic forms. Analytic and probabilistic methods in number theory. Proceedings of the 2nd international conference in honour of J. Kubilius, Palanga, Lithuania, September 2327, 1996. New trends in probability and statistics, Vol. 4, 35-43, VSP, Utrecht, 1997.

4. G. P. Gogishvili, On an estimate of singular series of positive quadratic forms. (Russian) Trudy Tbiliss. Univ. 346, Mat. Mekh. Astronom. (2003), 72-77 (2004).

5. I. Kiming, On certain problems in the analytical arithmetic of quadratic forms arising from the theory of curves of genus 2 with elliptic differentials. Manuscripta Math. 87(1995), No. 1, 101-129.

6. A. Malyshev, On the representation of integers by positive quadratic forms. (Russian) Trudy Mat. Inst. Steklov 65(1962), 212 pp.

7. R. A. Rankin, Sums of squares and cusp forms. Amer. J. Math. 87(1965), 857-860.

8. K. Prachar, Primzahlverteilung. Springer-Verlag, Berlin-Göttingen-Heidelberg, 1957.

(Received 21.09.2006)

Author's address:

Faculty of Exact and Natural Sciences

I. Javakhishvili Tbilisi State University

1, Chavchavadze Ave., Tbilisi 0128

Georgia

E-mail: guram@mzera.com 